\title{
Cellular automata-based model of group motion of agents with memory and related continuous model
}

\author{
Alexander V. Kuznetsov ${ }^{1}$ \\ ${ }^{1}$ Voronezh State University, 1 Universitetskaya pl., Voronezh, 394018, Russia
}

\begin{abstract}
The paper describes the construction of the motion and interaction model for agents with memory. Agents move on the landscape consisting of squares with different passability. We briefly characterize the cellular automata-based model with one common to all agents layer corresponding to the landscape and many agent-specific layers corresponding to an agent's memory. Also, we develop methods for the random landscape generation and the simulation of a communication system. Finally, we study a connection between the discrete agent motion model and the continuous concentration law for the system of agents.
\end{abstract}

Keywords: cellular automaton; motion model; conflict model; agent system; random landscape generation; landscape metrics; concentration law

\section{Introduction and definitions}

Previously the author studied cellular automaton-based models of motion [1] and communication [2]. The initial idea of the proposed model described in the article [3]. In this paper I continue the previous work, propose cellular automaton that takes into account the history of the movement of agents. Also, I obtain few quantitative characteristics of the model and found the continuous equation and corresponding problem for the partial differential equation which describes a dependence of the agents' number in the direction of agents motion on time and position. Note, that an automaton of the type mentioned above can be viewed as a 0 th order reflexive automaton [4].

Give definitions according to the work [5].

Definition 1. Let us call landscape $\mathcal{L}_{l}(n \times m)$ rectangle from $n \times m=N$ cells $\omega_{i j},(i, j) \in I \subset \mathbb{Z}^{2}$ with equal size belonging to $l$ different classes and that to $i$-th class it belongs $N_{i}$ cells, i.e. $\sum_{i=1}^{l} N_{i}=N$.

Note that for landscapes generated for testing of path-finding algorithms, landscape cells will be divided into classes according to the maximum possible cell-crossing speed.

Definition 2. Configuration entropy of the landscape $\mathcal{L}=\mathcal{L}_{l}(n \times m)$ is defined as

$$
S\left(\mathcal{L}_{l}(n \times m)\right)=-\sum_{i=1}^{l} \frac{N_{i}}{N} \ln \frac{N_{i}}{N}
$$

and characterizes landscape heterogeneity in whole.

Definition 3. Total Edge is the total number of abutting edges of cells, belonging to different classes, in $\mathcal{L}$. We will further denote the Total Edge of the landscape $\mathcal{L}$ as $T E(\mathcal{L})$.

Definition 4. Total Edge Density $(T E D)$ of the landscape $\mathcal{L}$ is the ratio $T E(\mathcal{L})$ to the total cell quantity $N$ in the $\mathcal{L}$

$$
\operatorname{TED}(\mathcal{L})=T E(\mathcal{L}) / N
$$

Definition 5. Denote Euclidean distance between $x=\left(x_{1}, \ldots, x_{n}\right), y=\left(y_{1}, \ldots, y_{n}\right), x, y \in \mathbb{R}^{n}$ as

$$
\|x-y\|=\left(\sum_{i=1}^{n}\left|x_{i}-y_{i}\right|^{2}\right)^{1 / 2} .
$$

\section{Description of the automaton}

Let the $A g=\left\{a g_{1}, \ldots, a g_{k}\right\}$ is the system of agents, which move across the landscape $\mathcal{L}_{l}(n \times m)$, and initial and final cells of the landscape are specified for each agent. The idea of the article is that in the model in addition to the total for all agents "layer" corresponding to objective reality, each agent would have been "layer" corresponding to the information about the reality, which is known to this agent.

The behavior of the agents of the system is modeled by a cellular automaton in which the set of cells is $W o r l d=\{(i, j, i d) \mid i, j \in$ $\mathbb{Z}, i d=\overline{0, k}\} \subset \mathbb{Z}^{3}$. In the set World we will allocate $k+1$ cell planes: a layer of the objective reality $O W o r l d=\{(i, j, 0) \mid i, j \in \mathbb{Z}\}$, and the layers of subjective reality of agent with identifier $a g=\overline{1, k} S \operatorname{World}_{a g}=\{(i, j, a g) \mid i, j \in \mathbb{Z}\}$. In this way,

$$
\text { World }=\text { OWorld } \cup\left(\bigcup_{a g=1}^{k} S \text { World } a g\right) .
$$

We assume that the rectangle $K \subset$ World, $K=\left\{(i, j, i d) \mid i=\overline{0, L_{K}}, j=\overline{0, L_{K}}, i d=\overline{0, k}\right\}$ is selected and all cells are in the resting state outside of it. 


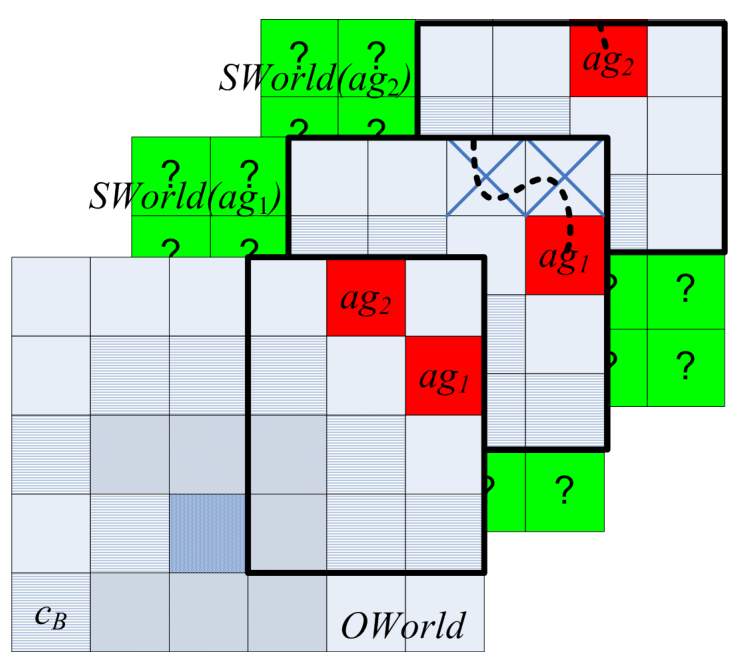

Fig. 1. The sample of the cellular automaton.

\subsection{Objective reality}

Let the objective reality layer OWorld consists of cells $(i, j) \in \mathbb{Z}^{2}$ with different impassability $u_{i j}$. The value of $u_{i j}$ is the number of discrete time units which is required to pass the square $\omega_{i j}$ with coordinates $(i, j)$. If $\omega_{i j}$ is completely impassable then put $u_{i j}=-1$. Also cells can include the information about an agent in a cell, the agent's destination square etc.

\subsection{Subjective reality}

The subjective reality layer consists of cells $(i, j)$ so that each its cell $(i, j)$ corresponds the cell $(i, j)$ of the objective reality layer. Cells of the subjective reality layer ag contain the information about the current position of the agent $a g$, about the history of the ag motion and about the impassability of known to the agent ag cells.

\subsection{The automaton's functioning}

Briefly describe the cellular automaton (CA) functioning. Let us denote

$$
\mathcal{D}=\{(-1,-1),(-1,0),(-1,1),(0,-1),(0,0),(0,1),(1,-1),(1,0),(1,1)\}
$$

Definition 6. Let us call agent's cellular route the sequence

$$
M=\left\{\left(i_{1}, j_{1}\right),\left(i_{2}, j_{2}\right), \ldots,\left(i_{s}, j_{s}\right) \mid\left(i_{k}, j_{k}\right) \in \mathbb{Z}^{2}, k=\overline{1, s},\left(i_{k+1}-i_{k}, j_{k+1}-j_{k}\right) \in \mathcal{D}, k=\overline{1, s-1}\right\},
$$

such as the agent in the square $\omega_{i_{1}, j_{1}}$ will be sequentially move into squares $\omega_{i_{2}, j_{2}}, \ldots, \omega_{i_{s}, j_{s}}$. Denote the set of all cellular routes starting in the cell with coordinates $c_{A} \in \mathbb{Z}^{2}$ and ending in the cell with coordinates $c_{B} \in \mathbb{Z}^{2}$ as $\mathscr{M}\left(c_{A} ; c_{B}\right)$.

Let us define a function

$$
\theta(x)= \begin{cases}0, & x<0 \\ 1, & x \geq 0\end{cases}
$$

Introduce the notation:

$$
\psi_{3}\left(u, T_{\max }\right)= \begin{cases}u, & u \geq 0, \\ T_{\max }, & u<0 .\end{cases}
$$

If cell impassability does not change over time, then it is not necessary to consider the routes containing impassable cells. However, if the impassable cell can become passable, these routes should be taken into account. To do this, define the functional

$$
\tilde{T}_{h}(M)=\sum_{(i, j) \in M}\left\|d_{i j}\right\| \psi_{3}\left(u_{i j}, T_{\max }\right) .
$$

We call weight of the route $M$ for the agent ag the following:

$$
\Lambda\left(M ; \alpha, \beta, \gamma, T_{\max }\right)=\alpha \tilde{T}_{h}(M)+\beta \sum_{(i, j) \in M} \theta\left(f_{i j}\right)+\gamma \sum_{(i, j) \in M} v i s_{i j}(a g)
$$

where $u_{i j}$ is the impassability of the square $\omega_{i j}$, vis $s_{i j}$ is the number of visits of the square $\omega_{i j}$ (it is contained in the subjective reality layer $S$ World $(a g)), \alpha, \beta, \gamma$ are parameters. 
The agent in the square $\omega_{i j}$ each discrete time tick tries to find locally optimal (in a neighborhood $V_{o}(i, j)$ with radius $o$ ) route $M_{o}$ such that $\Lambda\left(M_{o} ; \alpha, \beta, \gamma, T_{\max }\right) \rightarrow$ min and go through this route. Therefore, the agent's route at whole constructs from locally optimal subroutes.

Agent ag can apply previously described approach for route searching in undiscovered by this agent areas, i.e. in consisting of cells $\omega_{i j}$ with $v i s_{i j}(a g)=0$. More standard approaches to optimum route search, for example, Dijkstra's algorithm, can be used in areas composed of cells already visited. However, the use of standard methods of searching for an optimal route is limited to a rate of landscape change over time. It is possible that information about the visited cells become outdated (parameter $t_{i m e} e_{i j}(a g)$ is used for determining the actuality of information), or even impassibility of the cells would change directly during the process of passing the route selected as the globally optimal.

Thus, depending on the speed of the landscape changes, it is necessary to find a compromise between the approach "Reacting", which evaluates the current situation immediately near of the agent and the approach "Planning", in which searched globally optimal trajectory. For example, it is pointless to set the radius $o$ of the neighborhood $V_{o}(i, j)$, in which agent searches locally optimal route more than the number of ticks during which the landscape has remained unchanged.

The example of the described cellular automaton is depicted on the fig. 1. Increasing of the impassability at the mentioned figure is indicated with a darker tone, crosses " $X$ " in the layer $S$ World mark already visited cells, marks "?" correspond to cells whose status is unknown.

\section{Function of obstacles}

Turn to the continuous formulation of obstacle avoidance problem to construct transfer function for our CA. The agent moves in the domain $\Omega$ with changing over time obstacles from the point $A$ to the point $B$ with route $r(t), t \in[0, T]$ in the shortest time $T$. This problem has the form

$$
\begin{gathered}
\|\dot{r}(t)\|=v(t, r(t)), \\
r(0)=A, \quad r(T)=B, \\
T \rightarrow \min .
\end{gathered}
$$

We will call further the function $v:[0, T] \times \Omega \rightarrow \mathbb{R}$ as "function of obstacles". Divide the segment $[0, T]$ onto the $k$ subsegments with length $\tau>0$, domain $\Omega$ onto squares $\omega_{i j}$ with numbers $(i, j) \in \mathbb{Z}^{2}$ and the length of a side $h$. Approximate at each moment of time $k \tau \in[0, T]$ on the square $\omega_{i j}$ function $v(k \tau, \cdot)$ with the constant function $v_{i j}^{k}(h, \tau)$.

Go to the discrete time for the model simplifying. Let

If relations

$$
t_{h}=\frac{h}{\max _{(i, j) \in I_{h}, k \in T_{\tau}} v_{i j}^{k}(h, \tau)} .
$$

$$
u_{i j}(k)=\frac{h}{t_{h} v_{i j}^{k}(h, \tau)}=\frac{\max _{(i, j) \in I_{h}, k \in T_{\tau}} v_{i j}^{k}(h, \tau)}{v_{i j}^{k}(h, \tau)}
$$

hold, define that square $\omega_{i j}$ on the state in moment $k \tau \in[0, T]$ is crossable in non-diagonal direction in $u_{i j}(k)$ ticks.

Moreover, it is possible to go to the integer values of the $u_{i j}(k)$ by discarding the fractional part and taking $\tilde{u}_{i j}(k)=\left[u_{i j}(k)\right]$. We associate with the agent in the square $\omega_{i, j}$ value $\operatorname{errc}_{i j}$ of the cumulative discrete time error. Also we associate with the square $\omega_{i j}$ error value $\operatorname{err}_{i j}=\left\{u_{i j}(k)\right\}$. When an agent starts to cross the next square $\omega_{i^{\prime} j^{\prime}}$, the value $\operatorname{errc}_{i^{\prime} j^{\prime}}$ increments on the $\operatorname{err}_{i^{\prime} j^{\prime}}$, sets $\operatorname{errc}_{i j}=0$ and if $\operatorname{errc}_{i^{\prime} j^{\prime}}>1$ then agent passes one tick independently from the value of the function of obstacles in the square $\omega_{i^{\prime} j^{\prime}}$ and sets $\operatorname{errc}_{i^{\prime} j^{\prime}}=\operatorname{errc}_{i^{\prime} j^{\prime}}-1$.

Let

$$
T: \mathscr{M}\left(c_{A} ; c_{B}\right) \rightarrow \mathbb{R}
$$

the functional of time which is required to going through cellular route.

If values of the $u_{i j}$ do not change in a time of the movement from the point $A$ to the point $B$, then the problem (1)-(3) can be represented as discrete problem

$$
T(M)=t_{h} \sum_{(i, j) \in M}\left\|d_{i j}\right\| u_{i j} \rightarrow \min .
$$

It is clear that possible to minimize functional

$$
T_{h}(M)=\sum_{(i, j) \in M}\left\|d_{i j}\right\| u_{i j} \rightarrow \min
$$

instead functional $T$.

It is possibly (but not very easy) to prove that the CA mentioned earlier finds the approximation of the solution of the problem (1)-(3) in some subdomain of the $\Omega$. The sequence of such approximations $r_{h}$ converges to the optimal solution $r$, and the following estimate holds:

$$
\left|r(l(t))-r_{h}\left(l_{h}(t)\right)\right| \leq(h \sqrt{2}+\tau)\left(e^{\left\|\nabla_{(t, x, y)} v\right\|_{C([0, T] \times \Omega)} K}-1\right)+h \sqrt{2}
$$


where $l, l_{h}$ are parameterizations of routes, $h$ is the length of the square $\omega_{i j}$ side, $\tau$ is the length of the time tick, $K>0$ is the constant depending on a class of routes considered.

Definition 7. Define the obstacle which is exists in the moment $t \in[0, T]$ as simply connected set Obst $\subset \Omega$ such that any $r_{\text {obst }} \in$ Obst is the point of a local minimum of the function of obstacles $v(t, \cdot)$ and exists $r_{0} \in \Omega$ such that $v\left(t, r_{0}\right)>v\left(t, r_{\text {obst }}\right)$.

\section{The model of a communication system and conflict}

Definition 8. Let us define communication graph as follows

$$
\Gamma(t)=(A g, \operatorname{Comm}, \varphi(t), M(t)),
$$

where Comm is the set of channels, $\varphi(t): A g \times C o m m \rightarrow\{0,1\}$ is the incidence function, M(t) : Comm $\rightarrow \mathbb{R}^{n}$ is the markup function in the moment of time $t \in\left[0, N_{T}\right]$. The $M$ gives the features vector of the channel comm $\in$ Comm. This features can be channel bandwidth, radio frequency, etc.

Let's introduce a communication graph connected with the motion model. This means that should be given the function pag : $\left[0, N_{T}\right] \times A g \rightarrow \mathbb{Z}^{2}$ which maps agent's coordinates to an agent in each moment $t \in[0, T]$. Also, it means that $M(t)$ and $\varphi(t)$ depend on properties of cells in which incident agents are currently placed and on properties of cells between of them. Therefore, connections between agents in the $\Gamma$ can break and establish depending on the agents' speed and landscape type.

Suppose that each agent $a g \in A g$ has an own signal exchange timetable. It is possibly also to define specific signals like "enemy detecting", "grouping", etc. We can study various traffic models depending on the agents' timetables, motion speed, and the landscape type.

Finally, we can define "requirements graph" $\Pi$ and state that communication graph $\Gamma(t)$ should be similar with $\Pi$ in some metric each moment of time. Such graph $\Pi$ can be viewed as a fuzzy set of communication graphs, as an abstract container or as a generator of the stream of communication graphs.

Also, we developed the conflict model combined with the motion and communication model similar to described in the work [6] and its computer simulation "Bokohod." Agents emerge different kinds of tactics and exchange signals without any external control.

\section{Computational experiment}

Previously the author had developed the algorithm of the landscape generation with the given configuration entropy. This algorithm constructs the vector of numbers of cells in each class $V=\left(N_{1}, \ldots, N_{l}\right)$ by the given entropy $S$ as follows:

$\mathrm{S}$ t e p 1. Solve the equation

$$
S=-\frac{\beta\left(1-\beta^{l}\right)-(1-\beta) l \beta^{l}}{(1-\beta)\left(1-\beta^{l}\right)} \ln \beta+\ln \frac{1-\beta^{l}}{1-\beta},
$$

S te $\mathrm{p}$ 2. Use the found solution $0 \leq \beta \leq 1$ and equation

$$
N_{1}=N \frac{1-\beta}{1-\beta^{l}}
$$

to find $N_{1}$,

$\mathrm{S}$ t e p 3. Compose the vector $V_{0}=\left(N_{1}, \beta N_{1}, \ldots, \beta^{l-1} N_{1}\right)$,

$\mathrm{S}$ t e $\mathrm{p}$ 4. Round the components of the $V_{0}$ up to integers and obtain the vector $V_{1}$ in this way. It is necessary to make rounding such that the sum of all components of $V_{1}$ would be equal to $N$.

We generate landscape such as the discrete function of the obstacles $u: \mathbb{Z}^{2} \rightarrow \mathbb{Z}$ would have local maxima strictly in $N_{\text {obst }}=$ $V_{l}=\beta^{l-1} N_{1}$ cells. The author thinks that this method gives more natural-like landscapes as it makes "generally passable" area with some hardly passable subareas. As it known from [5] we can make very different landscapes with the same configuration entropy. By this reason, we will use the special, CA-based way of the filling landscape with cells of different classes. This method guarantees slow, near linear increasing of the TED at the increasing of the entropy.

Examples of obtained landscapes are shown on the fig. 2, (a,b). Sample dependencies of the configuration entropy, TED, and $N_{\text {obst }}$ are shown on the fig. 3 .

We set $l=9, n=m=48, o=6$, choose $N_{\text {obst }} \in\{5\} \cup\{10 i \mid i=\overline{1,25}\} \cup\{255\}$. Generate landscape for the each $N_{\text {obst }}$ value and perform the following experiment ${ }^{1}$. Let an agent moves from the cell $\omega_{11}$ to the cell $\omega_{n n} 100$ times according to the previously described algorithm. Next, we compute the time which is required for the experiment completion $T_{b o k}^{i}$ and the time of the moving from the $\omega_{11}$ to the $\omega_{n n}$ by linear straight route $T_{\text {tup }}^{i}$. Then we calculate the mean value and standard deviation of the win of time for all of this series:

$$
\overline{w i n}=\frac{1}{50} \sum_{i=1}^{50} \frac{T_{t u p}^{i}}{T_{b o k}^{i}} .
$$

\footnotetext{
${ }^{1}$ The raw data and the data processing program for all experiments are stored at https://www.researchgate.net/publication/316747096_The experimental_data_for_group_motion_of_agents_with_memory_with_the_program_in_Wolfram_Language.
} 


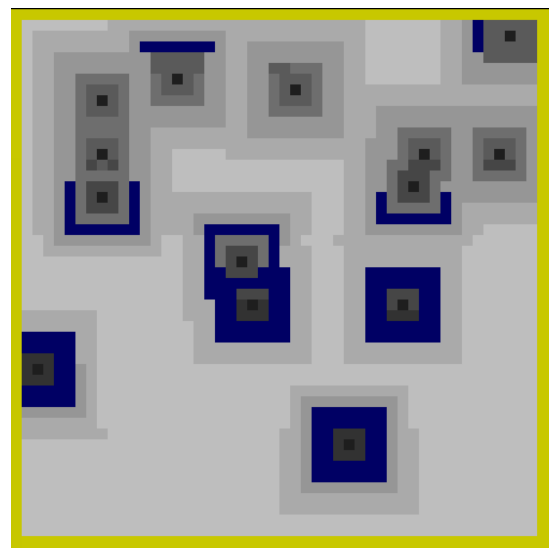

(a) $S=1.6, N_{\text {obst }}=14$

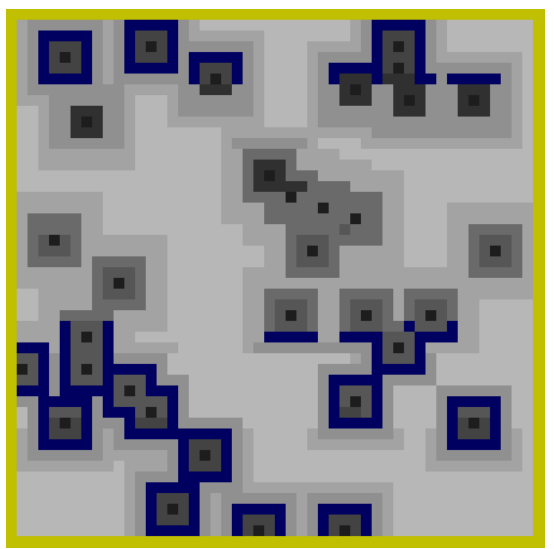

(b) $S=1.8, N_{\text {obst }}=33$

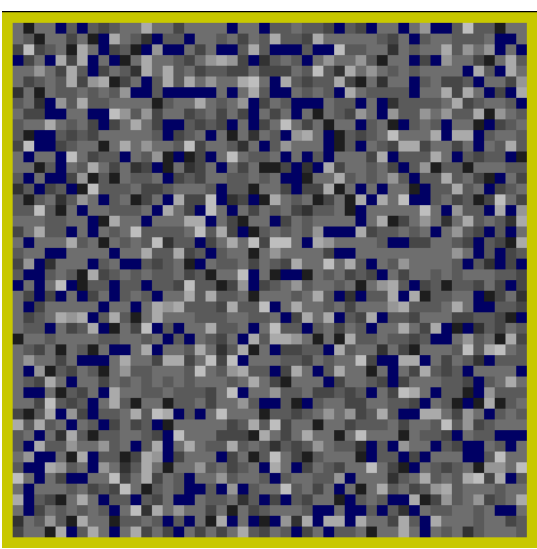

(c) $S=1.84877$

Fig. 2. Examples of Landscapes. A darker cell is more impassable.

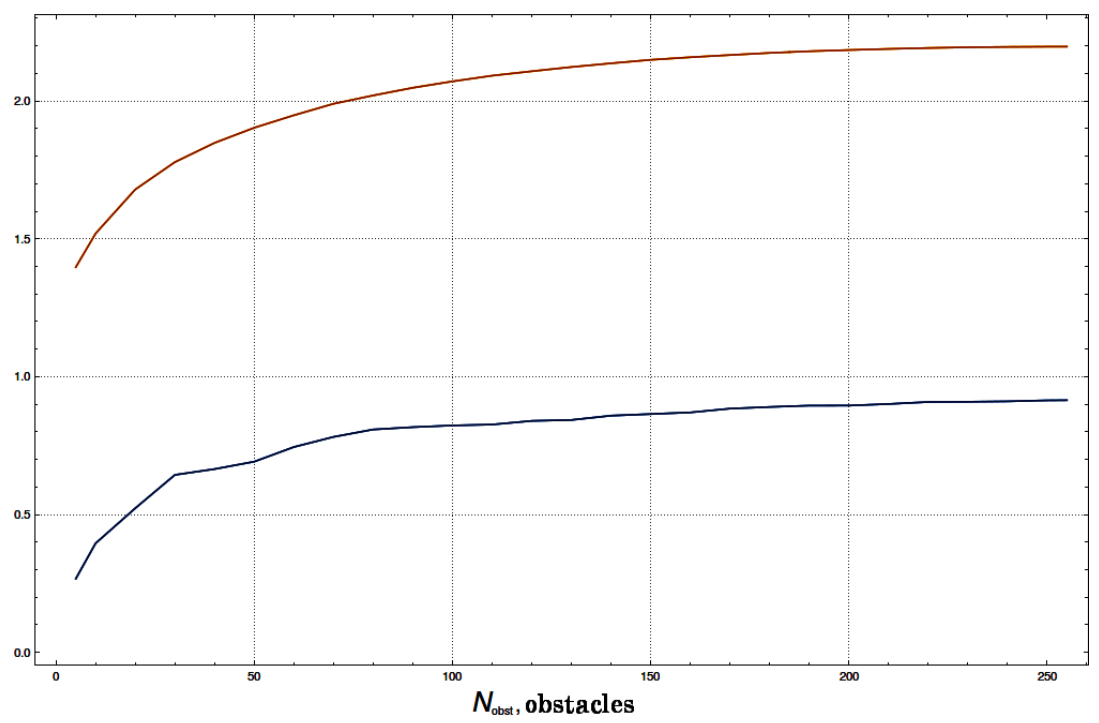

Fig. 3. Sample dependencies of the configuration entropy, TED and $N_{\text {obst }}$.

$$
\sigma=\left(\sum_{i=1}^{50}\left(\overline{w i n}-\frac{T_{t u p}^{i}}{T_{b o k}^{i}}\right)^{2}\right)^{1 / 2} .
$$

It was found that the mean value of the win in transit time $\overline{\text { win }}$ and the configuration entropy of the landscape $S$ are correlated with a correlation coefficient 0.959556 (see fig. 4). The $\overline{\text { win }}$ and the TED of the landscape are correlated with a correlation coefficient 0.964763 . The orange line in the figure corresponds to the curve $y=\left(S\left(N_{o b s t}\right)+1\right) \ln 9$; the brown line corresponds to the curve

$$
y=0.922178+0.539383 T E D\left(N_{\text {obst }}\right),
$$

where $S\left(N_{o b s t}\right)$ and $T E D\left(N_{o b s t}\right)$ are the entropy and the total edge density's mean value of the landscape with $N_{o b s t}$ obstacles. Vertical bars correspond to the standard deviation of the win of time.

Let's study the dependence of the average number of agents on time moment and position on a landscape. We generate 150 random landscapes with the given entropy by the algorithm mentioned above. The group formed from $u_{0}=48$ agents moves from the one side of the squared landscape to the opposite one. Compute dependence of the average (through all landscapes generated) number of agents $u$ at the $x$ th line of a landscape on the discrete time $t, x=\overline{1, x_{\max }}, x_{\max }=48$. Thus, we find that this dependence is determined, mainly, not by the particular kind of landscape, but by the landscape configuration entropy $S$. We found the dependence in the form

$$
u(x, t)=\frac{u_{0}}{2}\left(\operatorname{erf}\left(\xi_{1}(S ; x, t)\right)-\operatorname{erf}\left(\xi_{2}(S ; x, t)\right)\right)
$$

where

$$
\begin{gathered}
\xi_{1}(S ; x, t)=\frac{a(S ; x)}{\sqrt{t}}+b_{1}(S ; x), \\
\xi_{2}(S ; x, t)=\frac{a(S ; x)}{\sqrt{t}}+\operatorname{sgn}(48-x) b_{2}(S ; x),
\end{gathered}
$$




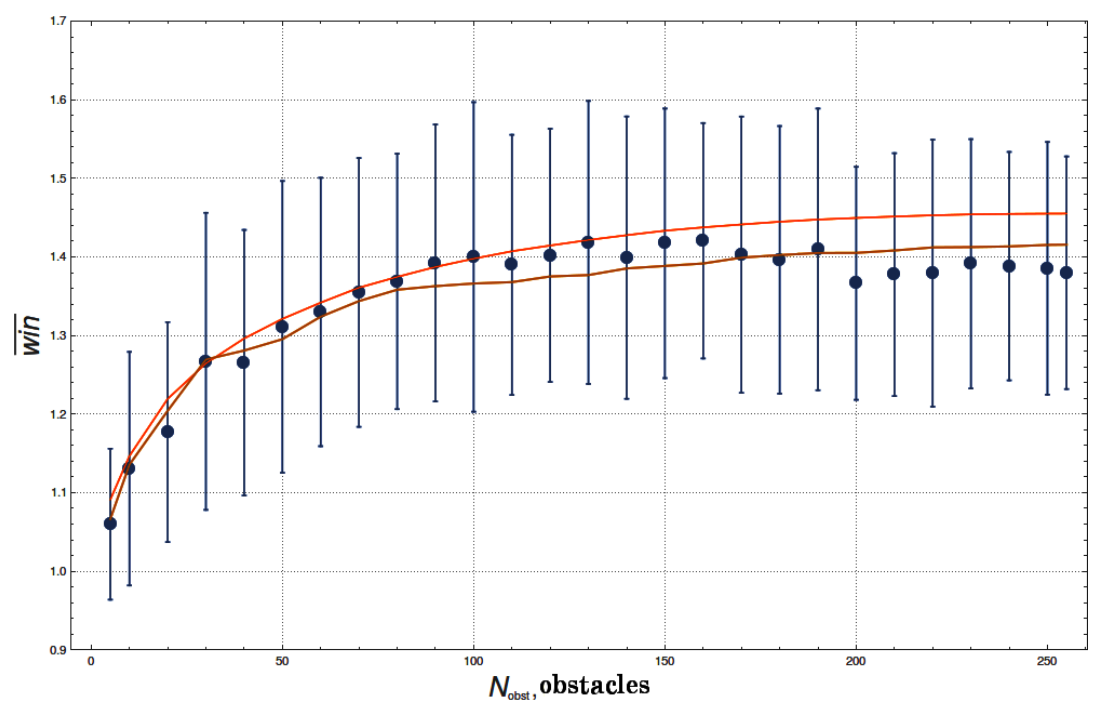

Fig. 4. Win of the time.

$$
\operatorname{sgn}(x)= \begin{cases}1, & x>0, \\ -1, & x \leq 0 .\end{cases}
$$

This form of $\xi_{1}$ and $\xi_{2}$ parameters was assumed by the analogy with the problem of heat propagation along a rod with a thermal diffusivity $a$ heated on its segment $\left[l_{1}, l_{2}\right]$ to the temperature $u_{0}$. This problem has (see, for example, [7]) solution

$$
u(x, t)=\frac{u_{0}}{2}\left(\operatorname{erf}\left(\frac{x-l_{1}}{2 a \sqrt{t}}\right)-\operatorname{erf}\left(\frac{x-l_{2}}{2 a \sqrt{t}}\right)\right)
$$

Let us assume that

$$
\begin{gathered}
a(S ; x)=a_{1}(S) x+a_{2}(S), \\
b_{1}(S ; x)=b_{11}(S) \sqrt{x}+b_{12}(S)+\frac{b_{13}(S)}{x^{3 / 2}}, \\
b_{2}(S ; x)=b_{11}(S) \sqrt{x}+b_{22}(S) .
\end{gathered}
$$

These functions allow to approximate $u=u(x, t)$ with the coefficient of determination $r^{2}>0.97$, the mean absolute error MAE $<$ 0.1251 , and the median absolute error $M e d A E<0.04366$ with every value of the entropy $S$. Experimental and approximated values of $u$ are shown on the fig. 5. For example, when $N_{\text {obst }}=20(S=1.67909)$

$$
\begin{array}{r}
u(x, t)=24\left(\operatorname{erf}\left(\frac{-2.04693 x-1.03949}{\sqrt{t}}+\frac{4.66438}{x^{3 / 2}}+1.08597 \sqrt{x}+0.926477\right)-\right. \\
\left.-\operatorname{erf}\left(\frac{-2.04693 x-1.03949}{\sqrt{t}}+1.08597 \sqrt{x}+\operatorname{sgn}(48-x) 0.845118\right)\right)
\end{array}
$$

Next, we try to find a problem for a partial differential equation which can have a solution in the form (5). Naturally, we can assume that this equation is

the initial condition is

$$
\frac{\partial u}{\partial t}=C_{1} \frac{\partial^{2} u}{\partial x^{2}}+C_{2} \frac{\partial u}{\partial x}
$$

$$
\begin{aligned}
& u(x, 0)=u_{0} \delta(x-1), \\
& \delta(x)= \begin{cases}1, & x=0, \\
0, & x \neq 0,\end{cases}
\end{aligned}
$$

and the asymptotic condition is

$$
\begin{gathered}
\lim _{t \rightarrow \infty}(x, t)=u_{0} \theta\left(x-x_{\max }\right), \\
\theta(x)= \begin{cases}1, & x \geq 0 \\
0, & x<0,\end{cases}
\end{gathered}
$$

Let us denote

$$
\begin{gathered}
U_{1}(x, t)=\mathrm{e}^{-\left(\frac{a(S ; x)}{\sqrt{t}}+b_{22}(S)+b_{11}(S) \sqrt{x}\right)^{2}}, \\
U_{2}(x, t)=\mathrm{e}^{-\left(\frac{a(S ; x)}{\sqrt{t}}+b_{11}(S) \sqrt{x}+b_{12}(S)+\frac{b_{13}(S)}{x^{3 / 2}}\right)^{2}} .
\end{gathered}
$$




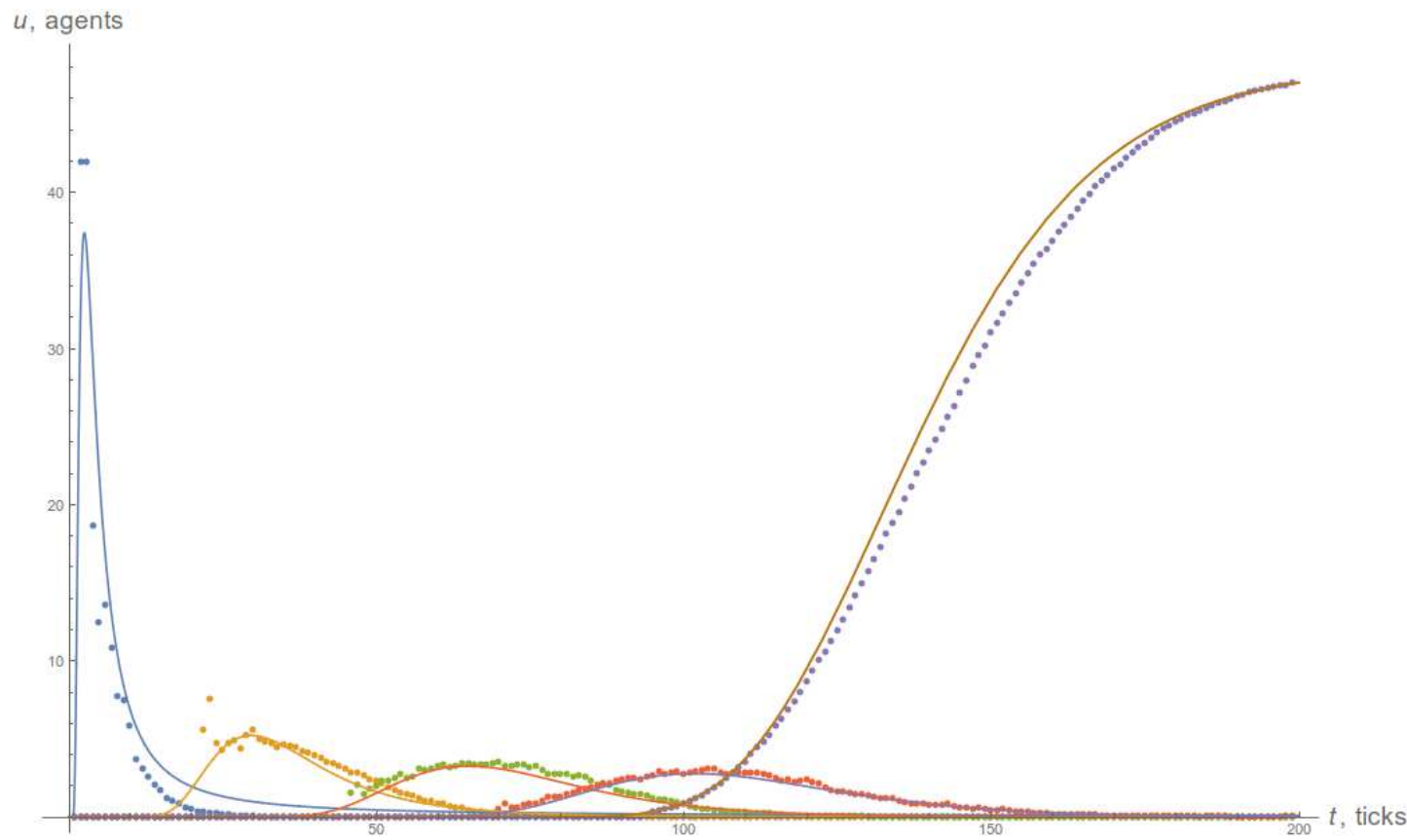

Fig. 5. The dependence of the average number of agents $u$ on the discrete time $t, N_{\text {obst }}=20, x=2, x=12, x=24, x=36$, and $x=48$ from the left to right.

Compute from the (5) that when $x \in\left(1, x_{\max }\right)$

$$
\begin{gathered}
\frac{\partial u}{\partial t}(x, t)=\frac{u_{0}\left(U_{1}(x, t)-U_{2}(x, t)\right)\left(a_{1}(S) x+a_{2}(S)\right)}{2 \sqrt{\pi t^{3}}} \\
\frac{\partial u}{\partial x}(x, t)=-\frac{u_{0}\left(x^{2}\left(U_{1}(x, t)-U_{2}(x, t)\right)\left(2 a_{1}(S) \sqrt{x}+b_{11}(S) \sqrt{t}\right)+3 b_{13}(S) \sqrt{t} U_{2}(x, t)\right)}{2 \sqrt{\pi t x^{5}}},
\end{gathered}
$$

$$
\begin{array}{r}
\frac{\partial^{2} u}{\partial x^{2}}(x, t)=\frac{u_{0}}{4 \sqrt{\pi} t^{3 / 2} x^{13 / 2}}\left(b _ { 1 3 } ( S ) \sqrt { t } U _ { 2 } ( x , t ) x ^ { 3 } \left(4\left(4 a_{1}(S)^{2} x^{2}+6 a_{1}(S) a_{2}(S) x+7 a_{1}(S) b_{11}(S) \sqrt{t x^{3}}+3 a_{2}(S) b_{11}(S) \sqrt{t x}\right)+\right.\right. \\
\left.\quad+24 a_{1}(S) b_{12}(S) \sqrt{t} x+t\left(10 b_{11}(S)^{2} x+12 b_{11}(S) b_{12}(S) \sqrt{x}+15\right)\right)+8 a_{1}(S)^{2} \sqrt{t x^{13}}\left(b_{22}(S) U_{1}(x, t)-b_{12}(S) U_{2}(x, t)\right)- \\
-6 b_{13}(S)^{2} t U_{2}(x, t) x\left(-a_{1}(S) x^{3 / 2}+3 a_{2}(S) \sqrt{x}+b_{11}(S) \sqrt{t} x+3 b_{12}(S) \sqrt{t x}\right)+8 a_{1}(S) b_{11}(S) t x^{6}\left(b_{22}(S) U_{1}(x, t)-b_{12}(S) U_{2}(x, t)\right)+ \\
+10 a_{1}(S) b_{11}(S)^{2} t U_{1}(x, t) x^{13 / 2}-10 a_{1}(S) b_{11}(S)^{2} t U_{2}(x, t) x^{13 / 2}+2 b_{22}(S) b_{11}(S)^{2} t^{3 / 2} U_{1}(x, t) x^{11 / 2}-2 b_{11}(S)^{2} b_{12}(S) t^{3 / 2} U_{2}(x, t) x^{11 / 2}- \\
-18 b_{13}(S)^{3} t^{3 / 2} U_{2}(x, t)+\left(U_{1}(x, t)-U_{2}(x, t)\right)\left(8 a_{1}(S)^{3} x^{15 / 2}+8 a_{1}(S)^{2} a_{2}(S) x^{13 / 2}+8 a_{1}(S) b_{11}(S) \sqrt{t} x^{6}\left(2 a_{1}(S) x+a_{2}(S)\right)+\right. \\
\left.+2 a_{2}(S) b_{11}(S)^{2} t x^{11 / 2}+2 b_{11}(S)^{3} t^{3 / 2} x^{6}+b_{11}(S) t^{3 / 2} x^{5}\right) . \quad(8)
\end{array}
$$

Solve (7), (8) with respect to $U_{1}, U_{2}$. Substitute into (6) values found and obtain

$$
C_{1}(x, t)=\frac{P_{1}(\sqrt{x}, \sqrt{t})}{Q_{1}(\sqrt{x}, \sqrt{t})}, \quad C_{2}(x, t)=\frac{P_{2}(\sqrt{x}, \sqrt{t})}{Q_{2}(\sqrt{x}, \sqrt{t})},
$$

where

$$
\begin{aligned}
Q_{1}(\sqrt{x}, & \sqrt{t})=-3 b_{11}(S) t x^{2}\left(4 a_{1}(S)^{2} x^{13 / 2}\left(b_{12}(S)-b_{22}(S)\right)-2 b_{13}(S) x^{3}\left(2 a_{1}(S) x\left(a_{1}(S) x+a_{2}(S)\right)-2 a_{1}(S) \sqrt{t} x\left(b_{22}(S)-2 b_{12}(S)\right)+t\right)+\right. \\
+ & \left.b_{13}(S)^{2}\left(a_{1}(S) \sqrt{t x^{5}}+3 a_{2}(S) \sqrt{t x^{3}}+3 b_{12}(S) t x^{3 / 2}\right)+3 b_{13}(S)^{3} t\right)+a_{1}(S)\left(8 a_{1}(S)^{2} \sqrt{t} x^{9}\left(b_{22}(S)-b_{12}(S)\right)+\right. \\
& +b_{13}(S)\left(4 a_{1}(S)\left(a_{1}(S) \sqrt{t x^{15}}+3 a_{2}(S) \sqrt{t x^{13}}\right)-12 a_{1}(S) t x^{13 / 2}\left(b_{22}(S)-2 b_{12}(S)\right)+15 t^{3 / 2} x^{11 / 2}\right)- \\
- & \left.6 b_{13}(S)^{2} t x^{4}\left(-a_{1}(S) x+3 a_{2}(S)+3 b_{12}(S) \sqrt{t}\right)-18 b_{13}(S)^{3} t^{3 / 2} x^{5 / 2}\right)+3 b_{11}(S)^{2} t^{3 / 2} x^{4}\left(b _ { 1 3 } ( S ) \left(3 a_{1}(S) x^{5 / 2}+a_{2}(S) x^{3 / 2}-\right.\right. \\
- & \left.\left.\left(b_{22}(S)-2 b_{12}(S)\right) \sqrt{t x^{3}}\right)+2 a_{1}(S) x^{4}\left(b_{22}(S)-b_{12}(S)\right)-b_{13}(S)^{2} \sqrt{t}\right)+b_{11}(S)^{3} t^{2} x^{6}\left(x^{3 / 2}\left(b_{22}(S)-b_{12}(S)\right)+2 b_{13}(S)\right)
\end{aligned}
$$




$$
\begin{aligned}
& Q_{2}(\sqrt{x}, \sqrt{t})=2 \sqrt{t}\left(-3 b_{11}(S) t x^{2}\left(4 a_{1}(S)^{2} x^{13 / 2}\left(b_{12}(S)-b_{22}(S)\right)-2 b_{13}(S) x^{3}\left(2 a_{1}(S) x\left(a_{1}(S) x+a_{2}(S)\right)-\right.\right.\right. \\
& \left.\left.-2 a_{1}(S) \sqrt{t} x\left(b_{22}(S)-2 b_{12}(S)\right)+t\right)+b_{13}(S)^{2}\left(a_{1}(S) \sqrt{t x^{5}}+3 a_{2}(S) \sqrt{t x^{3}}+3 b_{12}(S) t x^{3 / 2}\right)+3 b_{13}(S)^{3} t\right)+ \\
& +a_{1}(S)\left(8 a_{1}(S)^{2} \sqrt{t} x^{9}\left(b_{22}(S)-b_{12}(S)\right)+b_{13}(S)\left(4 a_{1}(S)\left(a_{1}(S) \sqrt{t x^{15}}+3 a_{2}(S) \sqrt{t x^{13}}\right)-\right.\right. \\
& \left.\left.-12 a_{1}(S) t x^{13 / 2}\left(b_{22}(S)-2 b_{12}(S)\right)+15 t^{3 / 2} x^{11 / 2}\right)-6 b_{13}(S)^{2} t x^{4}\left(-a_{1}(S) x+3 a_{2}(S)+3 b_{12}(S) \sqrt{t}\right)-18 b_{13}(S)^{3} t^{3 / 2} x^{5 / 2}\right)+ \\
& +3 b_{11}(S)^{2} t^{3 / 2} x^{4}\left(b_{13}(S)\left(3 a_{1}(S) x^{5 / 2}+a_{2}(S) x^{3 / 2}-\left(b_{22}(S)-2 b_{12}(S)\right) \sqrt{t x^{3}}\right)+2 a_{1}(S) x^{4}\left(b_{22}(S)-b_{12}(S)\right)-b_{13}(S)^{2} \sqrt{t}\right)+ \\
& \left.+b_{11}(S)^{3} t^{2} x^{6}\left(x^{3 / 2}\left(b_{22}(S)-b_{12}(S)\right)+2 b_{13}(S)\right)\right)
\end{aligned}
$$

$$
P_{1}(\sqrt{x}, \sqrt{t})=-3 b_{13}(S) \sqrt{t x^{13}}\left(a_{1}(S) x+a_{2}(S)\right)
$$

$$
\begin{array}{r}
P_{2}(\sqrt{x}, \sqrt{t})=x^{5 / 2}\left(a_{1}(S) x+a_{2}(S)\right)\left(-b_{13}(S)\left(4\left(4 a_{1}(S)^{2} x^{5}+6 a_{1}(S) a_{2}(S) x^{4}+7 a_{1}(S) b_{11}(S) \sqrt{t x^{9}}+3 a_{2}(S) b_{11}(S) \sqrt{t x^{7}}\right)+\right.\right. \\
\left.+24 a_{1}(S) b_{12}(S) \sqrt{t} x^{4}+t x^{3}\left(10 b_{11}(S)^{2} x+12 b_{11}(S) b_{12}(S) \sqrt{x}+15\right)\right)+6 b_{13}(S)^{2}\left(-a_{1}(S) \sqrt{t x^{5}}+3 a_{2}(S) \sqrt{t x^{3}}+b_{11}(S) t x^{2}+3 b_{12}(S) t x^{3 / 2}\right)- \\
\left.-2 x^{11 / 2}\left(b_{22}(S)-b_{12}(S)\right)\left(2 a_{1}(S) \sqrt{x}+b_{11}(S) \sqrt{t}\right)^{2}+18 b_{13}(S)^{3} t\right) .
\end{array}
$$

The form of coefficients $a_{1}(S), a_{2}(S), b_{11}(S), b_{12}(S), b_{13}(S), b_{22}(S)$ depends on the landscape generation way. If we generate landscape as described before then we can approximate these parameters as follows

$$
\begin{gathered}
a(S)=\alpha(S) a(20), \\
b_{1}(S ; x)=\beta_{1}(S) b_{1}(20 ; x), \\
b_{2}(S ; x)=\beta_{2}(S) b_{2}(20 ; x),
\end{gathered}
$$

where

$$
\begin{gathered}
\alpha(S)=0.000344002 \frac{N_{o b s t}(S)}{10}+1.00018, \quad M A E<0.001 \\
\beta_{1}(S)=\beta_{2}(S)=1.0582-0.0581965 \sqrt{\frac{N_{o b s t}(S)}{10}-1}, \quad M A E<0.005
\end{gathered}
$$

Results of such approximation are shown into the fig. $6, x=x_{\max }=48$.

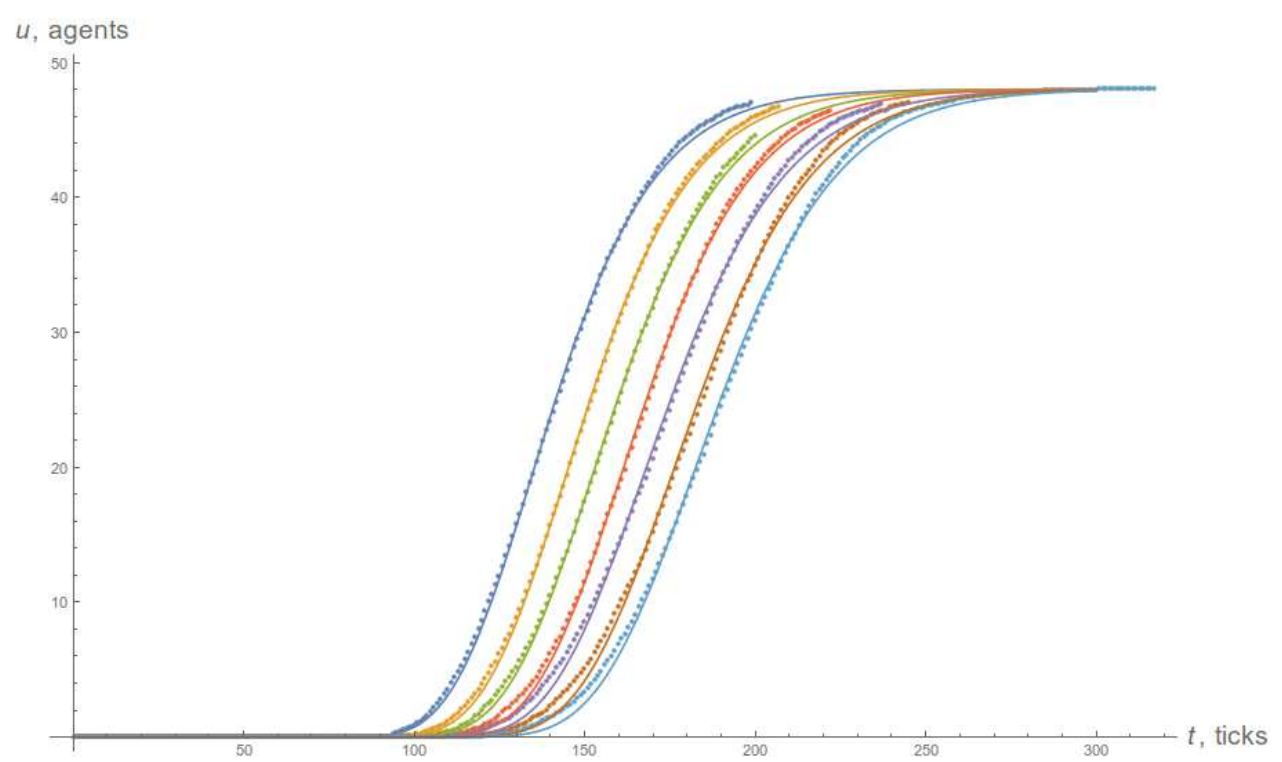

Fig. 6. The dependence $u$ on the entropy $S$, from the left to the right $N_{\text {obst }}=20, N_{\text {obst }}=40, N_{\text {obst }}=60, N_{\text {obst }}=80, N_{\text {obst }}=100, N_{\text {obst }}=120, N_{\text {obst }}=140$.

Note that we obtain different results by different landscape generation procedures. The general form of the function $u$ will be still described by the (5), but exact values of parameters can be entirely different. For example, it is possible to place squares of different classes on the uniformly random way (fig. 2c). The comparison of functions $u$ for a uniformly random landscape (right) and for landscape generated in the previously described way (left) are depicted on the fig. 7. 


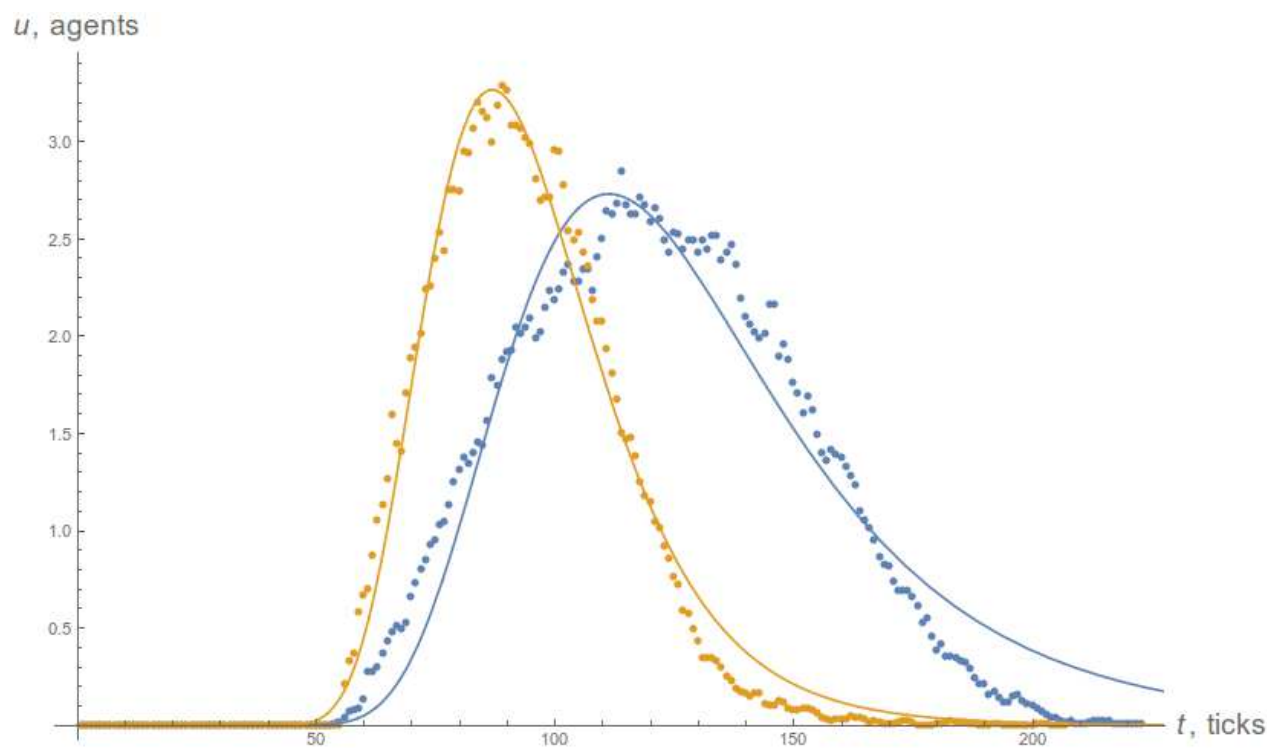

Fig. 7. Functions $u$ for differently generated landscapes, $x=26, S=1.84877$.

\section{Conclusion}

We obtained dependence of the win of movement by the proposed algorithm on the landscape's configuration entropy for some types of landscapes. The immediately following result may be a comparison of a model of the conflict based on the proposed cellular automaton with the result of solution of the corresponding Osipov-Lanchester equations. Also, we compare models of the "diffusion" of agents into a given sub-area based on the cellular automaton with the solution of the corresponding reaction-diffusion type equation. Finally, we can simulate the sharing of the subjective reality layers between agents. In this case, one agent will use the information about the area, received from other agents and will transmit such information to other agents itself. The algorithm described in the article can be applied to the mobile robot equipped with a transport base, navigation equipment (compass, GPS receiver, etc.), a sensor allowing to determine the impassibility of the terrain and the deciding unit, including memory.

\section{References}

[1] Kuznetsov, A. A model of the joint motion of agents with a three-level hierarchy based on a cellular automaton. Computational mathematics and mathematical physics 2017; 57(2): 340-349. [2] Kuznetsov A. Adaptive hierarchical system of the communication, control and decision support. Voronezh : NPF SAKVOEE LLC, 2016; 1: 269-277.

[3] Malinetskii GG, Stepantsov ME. Application of cellular automata for modeling the motion of a group of people. Computational Mathematics and Mathematical Physics $2004 ;$ 44(11): 1992-1996. [4] Schumann A. Payo cellular automata and reflexive games. J. Cellular Automata 2014; 9(4): 287-313.

[5] Cushman SA. Calculating the configurational entropy of a landscape mosaic. Landscape Ecology 2016; 31(3): 481-489. URL: http://dx.doi.org/10.1007/s10980-015-0305-2

[6] Ilachinski A. ArtificialWar: Multiagent-Based Simulation of Combat. Singapore : World Scientific Publishing Company, 2004; 747 p

[7] Tikhonov AN, Samarskii AA. Equations of Mathematical Physics. New York: Dover Publications, 1990; 800 p. 